\title{
Isolation of Klebsiella pneumoniae strains with altered susceptibility to carbapenems not carbapenemase mediated
}

Franca Cian', Maria Luisa Deiana', Clara Fabris', Anna Belgrano', Bruno Biasioli', Marco Maria D’Andrea², Tommaso Giani², Gian Maria Rossolini²

I Dipartimento di Medicina di Laboratorio - SC Microbiologia, Azienda Ospedaliero-Universitaria "Ospedali Riuniti" di Trieste

2 Dipartimento di Biologia Molecolare, Laboratorio Fi. Bi. M., Università degli Studi di Siena

Key words: Extended-spectrum B-lactamases (ESBL), Carbapenemase, Klebsiella pneumonia

Isolamento di ceppi di Klebsiella pneumoniae con alterata sensibilità ai carbapenemi non mediata da carbapenemasi

\section{SUMMARY}

The spread of enterobacteria producing extended-spectrum B-lactamases (ESBLs) is sharply increasing in Italy, while the detection of isolates resistant to carbapenems is still sporadic. Isolates of Klebsiella pneumoniae resistant to all cephalosporins, aminoglycosides and fluoroquinolones have been isolated in Trieste since 2008. Because of the altered profile of resistance to carbapenems, these strains were reported as ESBL-negative and possible carbapenemases producer by the expert system, leaving tigecycline as the only therapeutic choice. The purpose of this study is the characterization of the mechanisms involved in resistance to carbapenems in these strains and the evaluation of a reliable and simple test for phenotypic confirmation of ESBL and/or carbapenemase production.

25 isolates of MDR K. pneumoniae were collected between October 2008 and May 2009, mainly from urinary samples of elderly patients hospitalized in medicine wards. Identification and susceptibility testing were performed using the Vitek 2 system. The double-disc (DD) test was used to check the production of ESBLs, while imipenem and imipenem-EDTA synergy test was used to detect the production of metallo- $\beta$ lactamase (MBL). Carbapenemase activity was tested by an hydrolysis assay and the production of MBLs was also investigated by PCR.

The DD synergy test highlighted the possible production of ESBLs in 18 out of 22 strains, considered as negative by Vitek. All ESBLs

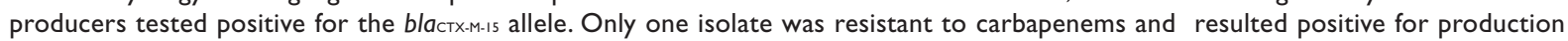
of MBL by the phenotypic test. The crude extract showed carbapenemase activity inhibited by EDTA; PCR test gave positive result for a bla VIM-type allele. PCR analysis performed on representative isolates, followed by sequencing, showed that coding sequence of ompk35 was not functional.

Results of this study confirmed the emergence of ESBL-positive strains of $K$. pneumoniae that showed altered sensitivity to carbapenems in absence of carbapenemases. For a correct identification of the resistance mechanisms of these isolates we used phenotypic tests (modified DD test and synergy test for MBL) coupled with molecular techniques. The MDR strains isolated in Trieste are particularly difficult in terms of therapeutic management, especially in case of at-risk subjects.

La diffusione di ceppi di enterobatteri resistenti ai ß-lattamici è in costante aumento in Europa; vari fattori sono chiamati in causa, ma il trasferimento di elementi di resistenza anche fra specie diverse gioca un ruolo rilevante soprattutto in ambiente ospedaliero dove l'uso esteso di terapie antibiotiche e il ritardo o la difficoltà nella rilevazione dei patogeni resistenti creano le condizioni ottimali alla propagazione di pericolosi cloni che rendono problematica la scelta terapeutica e aumentano la mortalità di pazienti in condizioni critiche.

Fra gli enterobatteri K. pneumoniae si è dimostrata negli ultimi anni una delle specie maggiormente in grado di acquisire determinanti di resistenza per molte classi di farmaci; in particolare sono in aumento esponenziale i ceppi produttori di ßlattamasi a spettro esteso (ESBLs) e sporadicamente si assiste alla comparsa di isolati resistenti anche ai carbapenemi. I dati sulla suscettibilità agli antibiotici in isolati di K. pneumoniae a Trieste si allineano a quelli degli stati europei con più alta frequenza di resistenza (1). In particolare si sono registrati un notevole incremento di ceppi resistenti alle cefalosporine di terza e quarta generazione dal 2006 al 2009 (Tabella 1) e l'improvvisa comparsa alla fine del 2008 di isolati resistenti anche ad aminoglicosidi e fluorochinoloni (multi drug resistant, MDR). L'elevato grado di resistenza alle cefalosporine di terza e quarta generazione e spesso la mancata rilevazione di ESBLs porta alla segnalazione, da parte del sistema esperto Vitek, di possibile produzione di carbapenemasi.

Questo studio si prefigge di valutare l'attendibilità del sistema automatizzato e di test manuali di conferma fenotipica nella rilevazione di ESBLs e carbapenemasi e di caratterizzare il meccanismo implicato nell'alterata sensibilità ai carbapenemici in questi ceppi MDR.
Tabella I. Resistenza agli antimicrobici in ceppi di K. pneumoniae isolati a Trieste. Sono riportate le percentuali di non sensibilità (intermedi + resistenti) dei ceppi di K. pneumoniae isolati tra il 2006 e il 2009 (*semestre gennaio-giugno).

AMC: Amoxicillina/Acido clavulanico; TZP: Piperacillina/Tazobactam; CTX: Cefotaxime; CAZ: Ceftazidime; FEP: Cefepime; IPM: Imipenem; AK: Amicacina; CN: Gentamicina; CIP: Ciprofloxacina

\begin{tabular}{lcccc}
\hline & $\begin{array}{c}\mathbf{2 0 0 6} \\
(\mathbf{n = 3 0 4})\end{array}$ & $\begin{array}{c}\mathbf{2 0 0 7} \\
(\mathbf{n = 3 0 2})\end{array}$ & $\begin{array}{c}\mathbf{2 0 0 8} \\
(\mathbf{n = 4 2 3})\end{array}$ & $\begin{array}{c}\mathbf{2 0 0 9} \\
(\mathbf{n = 1 9 2 *})\end{array}$ \\
\hline AMC & 6.90 & 5.28 & 30.5 & 39.06 \\
\hline TZP & 3.29 & 3.59 & 17.9 & 35.94 \\
\hline CTX & 5.75 & 5.28 & 27.42 & 34.90 \\
\hline CAZ & 6.25 & 5.80 & 27.83 & 34.90 \\
\hline FEP & 5.92 & 4.97 & 27.59 & 34.90 \\
\hline IPM & 0.66 & 0.00 & 0.94 & 0.00 \\
\hline AK & 0.33 & 1.66 & 7.08 & 15.63 \\
\hline CN & 0.99 & 3.31 & 10.14 & 16.67 \\
\hline CIP & 2.30 & 3.59 & 24.29 & 35.42 \\
\hline
\end{tabular}

Nel periodo ottobre 2008 - maggio 2009 sono stati isolati 25 ceppi di K. pneumoniae MDR in prevalenza da campioni urinari $(n=23)$, ma anche da emocoltura $(n=1)$ e broncoaspirati $(\mathrm{n}=1)$, in reparti medici o geriatrici. Identificazione e antibiogramma sono stati eseguiti utilizzando il sistema Vitek 2 (software Advanced Expert System 4.01), card GN, ASTN097 e AST-GN14 (bioMérieux, Roma, Italia).

La produzione di ESBLs è stata verificata con il test del doppio disco (DD): su Agar Mueller-Hinton, inoculato con una sospensione batterica a densità $0.5 \mathrm{McF}$ arland, sono stati posizionati dischetti di aztreonam (ATM $30 \mu \mathrm{g}$ ), ceftazidime (CAZ $30 \mu \mathrm{g}$ ), ceftriaxone (CRO $30 \mu \mathrm{g}$ ), cefepime (FEP 30 $\mu \mathrm{g}$ ), cefoxitina (FOX $30 \mu \mathrm{g})$, cefotaxime (CTX $30 \mu \mathrm{g}$ ) e al

\section{Corresponding author: Franca Cian}

SC Microbiologia, Azienda Ospedaliero-Universitaria "Ospedali Riuniti" di Trieste

34100 Trieste, Italy - Tel.: 040 3994346; Fax 0403994746

E-mail: franca.cian@aots.sanita.fvg.it 
centro della piastra un dischetto di amoxicillina-acido clavulanico (AMC $30 \mu \mathrm{g}$ ); l'aumento e/o la deformazione dell'area di inibizione della crescita verso AMC, come pure la presenza di una zona "fantasma", sono stati considerati indice di produzione ESBL. Per controllare la produzione di metallo ß-lattamasi (MBLs) è stato eseguito il test di sinergia tra imipenem (IPM $10 \mu \mathrm{g}$ ) e IPM $+8 \mu \mathrm{l}$ di EDTA $0.25 \mathrm{M}$; una differenza $>7 \mathrm{~mm}$ fra il diametro di inibizione ottenuto con IPM e quello di IPM+EDTA può indicare produzione di MBL (2).

La sensibilità alla tigeciclina è stata controllata con E-test (AB Biodisk, Solna, Sweden) seguendo le istruzioni della ditta produttrice. Per i criteri interpretativi sono state seguite le linee guida del Clinical and Laboratory Standards Institute (3). Come controllo di qualità è stato usato Escherichia coli ATCC 25922.

L'attività idrolitica nei confronti dei carbapenemi è stata saggiata utilizzando estratti grezzi degli isolati ed imipenem come substrato, secondo il protocollo descritto da Lauretti e colleghi (4).

La presenza di alleli codificanti per le ß-lattamasi di tipo bla $_{\text {стх-м, }}$ bla $a_{\text {ум }}$ e bla $a_{\text {Iмр }}$ è stata indagata tramite metodica PCR, come precedentemente descritto $(5,6)$.

Alterazioni nella produzione delle porine ompK35 e ompK36 sono state investigate tramite amplificazione delle sequenze codificanti e successivo sequenziamento (7). La clonalità degli isolati è stata indagata tramite metodica Multi-Locus Sequence Typing (MLST) secondo lo schema proposto da Diancourt e colleghi (8). Tutti gli amplificati ottenuti sono stati sequenziati da una ditta esterna (Macrogen Inc., Seoul, Korea), quando necessario.

\section{Antibiogramma automatizzato}

Tra i ceppi analizzati tramite il sistema automatizzato, ne sono stati scelti cinque, rappresentativi dei 25 isolati MDR, i cui risultati sono riassunti nella Tabella 2.

Tutti i ceppi risultano inoltre resistenti con MIC elevate ai fluorochinoloni (norfloxacina, ciprofloxacina e levofloxacina), al trimethoprim/sulfametoxazolo, nitrofurantoina e a piperacillina/tazobactam. Negli isolati 1, 3 e 4 entrambe le card non segnalano produzione di ESBLs; nell'isolato 2 la card GN14 rileva presenza di ESBLs non segnalata dalla card N097; l'isolato 5 è l'unico che risulta positivo con entrambe le card per la produzione di ESBLs. In tutti i ceppi il sistema automatizzato propone la modifica per imipenem da sensibi- le o intermedio a resistente e c'è concordanza tra i risultati della card AST-N097 e GN14. Similmente in tutti i ceppi, tranne il numero 4 , il sistema rileva sensibilità al meropenem e propone la modifica a R. Il ceppo 4 si differenzia dagli altri perché sensibile ad aminoglicosidi e aztreonam, intermedio a ceftriaxone, con alterata sensibilità a cefepime $(\mathrm{MIC}=8)$, inoltre presenta MIC insolitamente elevate per i carbapenemi $(\mathrm{EPM} \geq 8, \mathrm{IPM}=8$ e $\mathrm{MEM} \geq 16$ ).

Il ceppo 5 affianca al profilo di resistenza tipico anche l'alterata sensibilità alla tigeciclina, confermata con E-test $(\mathrm{MIC}=3, \mathrm{I})$.

\section{Caratterizzazione fenotipica e molecolare della produzio-} ne di ß-lattamasi

Ceppo 1: il test DD eseguito utilizzando un distributore di dischetti non rileva presenza di ESBL (Figura I a sinistra); l'utilizzo di dischetti di CAZ e FEP ad una distanza $\leq 20 \mathrm{~mm}$ da AMC permette di evidenziare una zona fantasma fra AMC e CAZ (Figura I a destra, freccia); l'EDTA, modificando la permeabilità di membrana, favorirebbe l'entrata dell'antibiotico e l'azione degli enzimi amplificando la deformazione dell'area di inibizione di crescita tipica della presenza di ESBL. Ceppo 2: il test DD a dischi ravvicinati conferma la presenza di ESBLs rilevata dalla card GN14; anche in questo caso l'EDTA amplifica l'area di inibizione (Figura II).

Ceppo 3: il test DD modificato non rileva segni indicativi di produzione di ESBLs. Si osserva anche in questo caso un'inibizione della crescita attorno ad AMC+EDTA che potrebbe essere imputata all'azione dell'EDTA sulla membrana (dati non mostrati).

Ceppo 4: il test DD modificato (Figura IIII a sinistra) evidenzia una netta differenza fra gli aloni di inibizione di AMC e AMC+EDTA, non imputabile solo all'azione dell'EDTA sulla membrana; il test di sinergia tra IPM e IPM + EDTA (Figura III a destra) rileva una differenza fra le zone di inibizione della crescita in assenza e presenza di EDTA superiore ai $7 \mathrm{~mm}$, indicativa della produzione di MBLs; la deformazione dell'anello di inibizione della crescita verso il cefepime (Figura III a sinistra in basso) potrebbe indicare anche la contemporanea produzione di ESBLs.

L'analisi PCR per le ß-lattamasi ha rilevato la presenza, in tutti gli isolati, dell'allele bla ${ }_{\mathrm{CTX}-\mathrm{M}-15}$. Tutti gli isolati presentano attività idrolitica per l'imipenem negativa tranne nel caso dell'isolato numero 4 , che è risultato poi essere positivo per un determinante di tipo blaviм. L'analisi MLST ha rilevato

Tabella 2. Risultati degli antibiogrammi automatizzati eseguiti su 5 ceppi rappresentativi di K. pneumoniae. Sono riportati solo parte degli antibiotici saggiati dalle card N097 e GNI4 Le modifiche proposte dal sistema esperto Vitek sono riportate in grassetto. ATM:Aztreonam; CAZ: Ceftazidime; CTX: Cefotaxime; CRO: Ceftriaxone; FEP: Cefepime; AMC:Amoxicillina/Acido clavulanico; EPM: Ertapenem; IPM: Imipenem; MEM: Meropenem;AK:Amicacina; CN: Gentamicina;TIG:Tigeciclina

\begin{tabular}{|c|c|c|c|c|c|c|c|c|c|c|c|c|c|c|c|c|c|}
\hline ceppi & Vitek & ATM & CAZ & CTX & CRO & \multirow{2}{*}{\multicolumn{2}{|c|}{$\begin{array}{c}\text { FEP } \\
\geq 64 \mathrm{R}\end{array}$}} & \multirow{2}{*}{$\begin{array}{l}\text { AMC } \\
\geq 32 \mathrm{R}\end{array}$} & \multirow[t]{2}{*}{ EPM } & \multicolumn{2}{|c|}{ IPM } & \multicolumn{2}{|c|}{ MEM } & \multirow{2}{*}{$\begin{array}{c}\text { ESBL } \\
\text { NEG }\end{array}$} & \multirow{2}{*}{$\frac{\text { AK }}{264 \mathrm{R}}$} & \multirow{2}{*}{$\frac{\mathrm{CN}}{216 \mathrm{R}}$} & \multirow{2}{*}{$\frac{\text { TIG }}{2 \mathrm{~S}}$} \\
\hline \multirow{2}{*}{1} & N097 & & $\geq 64 \mathrm{R}$ & $\geq 64 \mathrm{R}$ & & & & & & $\leq 1 \mathrm{~S}$ & $\geq 4 \mathrm{R}$ & $1 \mathrm{~S}$ & $1 R$ & & & & \\
\hline & GN14 & $\geq 64 \mathrm{R}$ & $\geq 64 \mathrm{R}$ & & $\geq 64 \mathrm{R}$ & $\geq 64$ & $4 \mathrm{R}$ & $\geq 32 \mathrm{R}$ & $\geq 8 \mathrm{R}$ & $\leq 1 \mathrm{~S}$ & $\geq 4 \mathrm{R}$ & $1 \mathrm{~S}$ & $1 R$ & NEG & $\geq 64 \mathrm{R}$ & $\geq 16 \mathrm{R}$ & \\
\hline \multirow{2}{*}{2} & N097 & & $\geq 64 \mathrm{R}$ & $264 \mathrm{R}$ & & $\geq 6$ & $4 R$ & $\geq 32 \mathrm{R}$ & & $\leq 1 \mathrm{~S}$ & $\geq 4 \mathrm{R}$ & $1 \mathrm{~S}$ & $1 R$ & & $\geq 64 \mathrm{R}$ & $\geq 16 \mathrm{R}$ & $2 \mathrm{~S}$ \\
\hline & GN14 & $\geq 64 \mathrm{R}$ & $\geq 64 \mathrm{R}$ & & $\geq 64 \mathrm{R}$ & $\geq 64$ & $4 \mathrm{R}$ & $\geq 32 \mathrm{R}$ & $\geq 8 \mathrm{R}$ & $\leq 1 \mathrm{~S}$ & $\geq 4 \mathrm{R}$ & $1 \mathrm{~s}$ & $1 R$ & 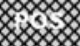 & $\geq 64 \mathrm{R}$ & $\geq 16 \mathrm{R}$ & \\
\hline \multirow{2}{*}{3} & N097 & & $\geq 64 \mathrm{R}$ & $\geq 64 \mathrm{R}$ & & $\geq 64$ & $4 R$ & $\geq 32 \mathrm{R}$ & & $\leq 1 \mathrm{~S}$ & $\geq 4 \mathrm{R}$ & $1 \mathrm{~S}$ & $1 R$ & NEG & $\geq 64 \mathrm{R}$ & $\geq 16 \mathrm{R}$ & $2 S$ \\
\hline & GN14 & $\geq 64 \mathrm{R}$ & $\geq 64 \mathrm{R}$ & & $\geq 64 \mathrm{R}$ & $\geq 64$ & $4 \mathrm{R}$ & $\geq 32 \mathrm{R}$ & $\geq 8 \mathrm{R}$ & $\leq 1 \mathrm{~S}$ & $\geq 4 \mathrm{R}$ & $1 \mathrm{~s}$ & $1 R$ & NEG & $\geq 64 \mathrm{R}$ & $\geq 16 \mathrm{R}$ & \\
\hline \multirow{2}{*}{4} & N097 & & $\geq 64 \mathrm{R}$ & $\geq 64 \mathrm{R}$ & & $8 \mathrm{~s}$ & $8 R$ & $\geq 32 \mathrm{R}$ & & 81 & $8 R$ & $\geq 16 \mathrm{R}$ & $\geq 16 \mathrm{R}$ & NEG & $\leq 2 \mathrm{~S}$ & $4 \mathrm{~S}$ & $1 \mathrm{~S}$ \\
\hline & GN14 & $\leq 1 \mathrm{~S}$ & $\geq 64 \mathrm{R}$ & & $\geq 64 \mathrm{R}$ & $8 \mathrm{~s}$ & $8 R$ & $\geq 32 \mathrm{R}$ & $\geq 8 \mathrm{R}$ & 81 & $8 R$ & $2 \mathrm{~S}$ & $2 R$ & NEG & $\leq 2 \mathrm{~S}$ & $4 \mathrm{~S}$ & \\
\hline \multirow{2}{*}{5} & N097 & & $\geq 64 \mathrm{R}$ & $\geq 64 \mathrm{R}$ & & $\geq 64$ & $4 R$ & $\geq 32 \mathrm{R}$ & & $\leq 1 \mathrm{~S}$ & $\geq 4 \mathrm{R}$ & $1 \mathrm{~s}$ & $1 R$ & POS & $\geq 64 \mathrm{R}$ & $\geq 16 \mathrm{R}$ & $\geq 8 \mathrm{R}$ \\
\hline & GN14 & $\geq 64 \mathrm{R}$ & $\geq 64 \mathrm{R}$ & & $\geq 64 \mathrm{R}$ & $\geq 64$ & $4 \mathrm{R}$ & $\geq 32 \mathrm{R}$ & $\geq 8 \mathrm{R}$ & $\leq 1 \mathrm{~S}$ & $\geq 4 \mathrm{R}$ & $1 \mathrm{~S}$ & $1 R$ & POS & $\geq 64 \mathrm{R}$ & $\geq 16 \mathrm{R}$ & \\
\hline
\end{tabular}


l'appartenenza di tutti gli stipiti studiati al Sequence Type 37. La permeabilità di membrana degli isolati risulta inoltre alterata a causa di una interruzione nella sequenza codificante la porina OmpK35.

I risultati del sistema automatico, utilizzando card diverse per la sensibilità agli antibiotici nei Gram negativi, sono sovrap-

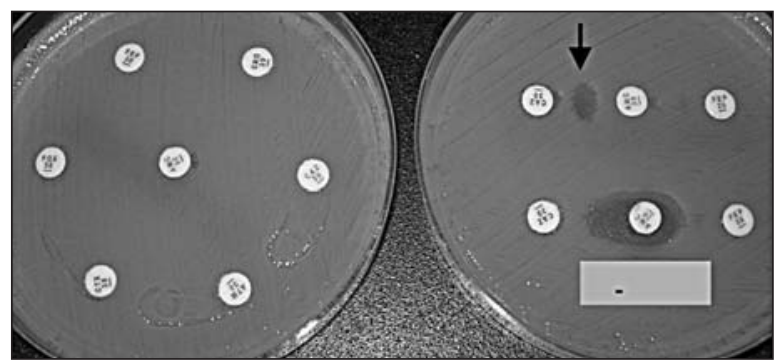

Figura I. Test $D D$ con distributore di dischetti (a sinistra) e test $D D$ manuale con dischetti a distanza ravvicinata (a destra) eseguiti inoculando l'isolato numero $I$.

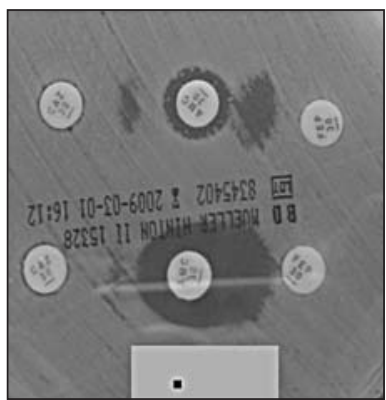

Figura II. Test DD manuale con dischetti a distanza ravvicinata sull'isolato numero 2

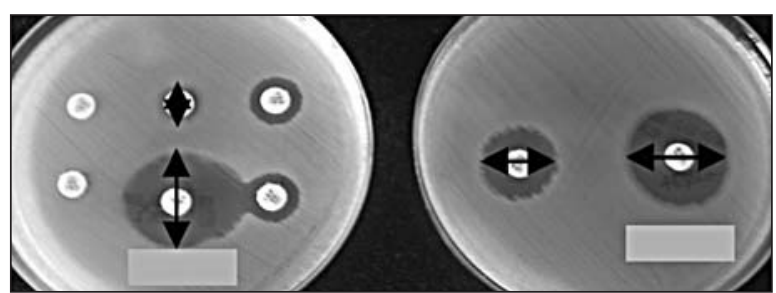

Figura III. Test DD modificato (a sinistra) e test di sinergia tra IPM e IPM + EDTA (a destra) sull'isolato numero 4 ponibili tranne nella rilevazione delle ESBL, che vengono evidenziate, solo dalla card GN14 e non dalla N097, in 3 isolati sui 12 testati.

Vitek (card N097); il test di sinergia con IPM e IPM+EDTA si conferma valido per la rilevazione dei ceppi produttori di MBLs; l'uso combinato di DD a dischi ravvicinati e di AMC+EDTA permette la contemporanea evidenziazione di ESBLs e MBLs.

La tecnica MLST ha evidenziato che gli isolati appartenevano al Sequence Type 37, come già riportato per altri isolati italiani con alterata sensibilità ai carbapenemi dovuta a perdita di porine (9). L'alterata sensibilità ai carbapenemi è risultata dovuta, per tutti gli isolati, tranne che per quello positivo per blaviм, ad una alterazione della permeabilità della membrana. La comparsa di K. pneumoniae con queste caratteristiche pregiudica il possibile utilizzo di carbapenemi nel trattamento di queste infezioni, restringendo notevolmente la scelta terapeutica.

\section{BIBLIOGRAFIA}

1. Coque TM, Baquero F, Canton R. Increasing prevalence of ESBL-producing Enterobacteriaceae in Europe. Eurosurveillance 2008; 13 Issue 47.

2. Drieux L, Brossier F, Sougakoff W, Jarlier V. Phenotipic detection of extended-spectrum $\beta$-lactamase production in Enterobacteriaceae: review and bench guide. Clin Microbiol Infect 2008; 14 (Suppl. 1): 90-103.

3. Clinical and Laboratory Standards Institute. 2009. Performance standards for antimicrobial susceptibility testing; $19^{\text {th }}$ informational supplement. CLSI documents M100-S19. Clinical and Laboratory Standards Institute, Wayne, $\mathrm{Pa}$.

4. Lauretti L, Riccio ML, Mazzariol A, et al. Cloning and characterization of blaVIM, a new integron-borne metallo-beta-lactamase gene from a Pseudomonas aeruginosa clinical isolate. Antimicrob Agents Chemother 1999; 43(7): 1584-90.

5. Luzzaro F, Docquier JD, Colinon C, et al. Emergence in Klebsiella pneumoniae and Enterobacter cloacae clinical isolates of the VIM-4 metallobeta-lactamase encoded by a conjugative plasmid. Antimicrob Agents Chemother 2004; 48(2): 648-50.

6. Pallecchi L, Malossi M, Mantella A, et al. Detection of CTX-M-type betalactamase genes in fecal Escherichia coli isolates from healthy children in Bolivia and Peru. Antimicrob Agents Chemother. 2004; 48(12): 4556-61.

7. Kaczmarek FM, Dib-Hajj F, Shang W, Gootz TD. High-level carbapenem resistance in a Klebsiella pneumoniae clinical isolate is due to the combination of bla(ACT-1) beta-lactamase production, porin OmpK35/36 insertional inactivation, and down-regulation of the phosphate transport porin phoe. Antimicrob Agents Chemother 2006; 50: 3396-406.

8. Diancourt L, Passet V, Verhoef J, Grimont PA, Brisse S. Multilocus sequence typing of Klebsiella pneumoniae nosocomial isolates. J Clin Microbiol. 2005; 43(8): 4178-82.

9. D'Andrea MM, Giani T, Arena F, et al. Multifocal emergence of ESBLproducing Klebsiella pneumoniae clone with differential non carbapenemase-mediated resistance to carbapenems in Italian hospitals. Poster P1007 ECCMID 2009 16-19 May Helsinki. 